$\frac{12}{8.495} 850$

UCRL-ID-120307

\title{
Interim Cryo-Cooler/Detector Report
}

\author{
K. Neufeld \\ W. Ruhter \\ E. Anderson
}

April 19, 1995

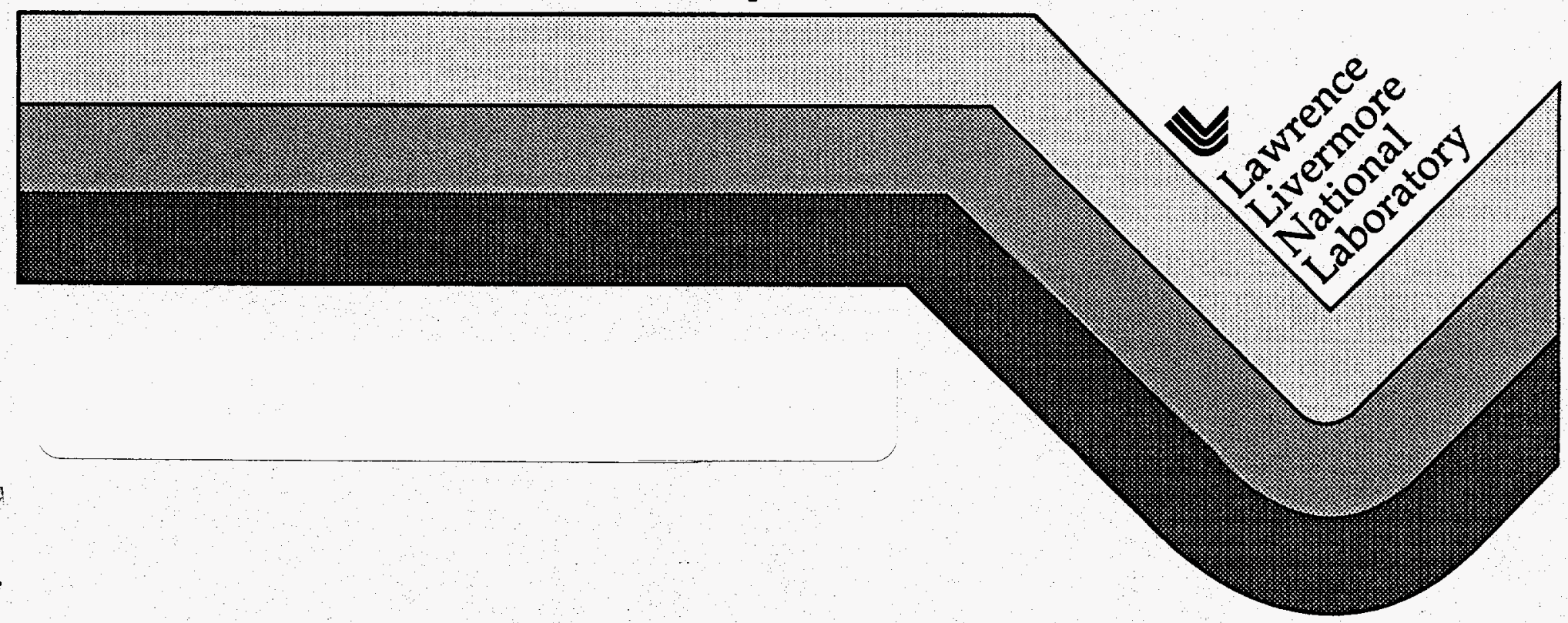


This document was prepared as an account of work sponsored by an agency of the United States Government. Neither the United States Government nor the University of California nor any of their employees, makes any warranty, express or implied, or assumies any legal liability or responsibility for the accuracy, completeness, or usefulness of any information, apparatus, product, or process disclosed, or represents that its use would not infringe privately owned rights. Reference herein to any specific commercial product, process, or service by trade name, trademark, mamufacturer, or otherwise, does not necessarily constitute or imply its endorsement, recommendation, or favoring by the United States Government or the University of California. The views and opinions of authors expressed herein do not necessarily state or reflect those of the United States Government or the University of California, and shall not be used for advertising or product endorsement purposes.

This report has been reproduced directly from the best available copy.

Available to DOE and DOE contractors from the Office of Scientific and Technical Information P.O. Box 62, Oak Ridge, TN 37831

Prices available from (615) 576-8401, FTS 626-8401

Available to the public from the National Technical Information Service

U.S. Department of Commerce 5285 Port Royal Rd.,

Springfield, VA 22161 


\section{DISCLAIMER}

Portions of this document may be illegible in electronic image products. Images are produced from the best available original document. 


\title{
Interim Cryo-Cooler/Detector Report
}

\author{
Kenneth W. Neufeld and Wayne D. Ruhter \\ Lawrence Livermore National Laboratory \\ Livermore, CA 94550 \\ and \\ Eric H. Anderson \\ CSA Engineering, Inc. \\ Palo Alto, CA 94303-3843
}

* Work performed under the auspices of the U.S. Department of Energy by the Lawrence Livermore National Laboratory under Contract W-7405-Eng-48. This work was supported by DOE's Office of Research and Development, NN-20. 


\section{EXECUTIVE SUMMMARY}

This report describes development of an electronic system designed to reduce vibration generated by a cryocooler. The diminished vibration makes it practical to use the active cooler to extract heat from a portable gamma ray detector instrument. The system was developed for a Sunpower cryocooler with an integrated counterbalance mass. The overall momentum cancellation approach is also applicable to other similar cryocoolers.

The cancellation system is an assembly of several components tailored to accomplish the required vibration reduction with minimum power consumption and volume. It is designed to be powered by a 18--32 Volt battery. Up to ten harmonics of the $58.65 \mathrm{~Hz}$ drive frequency are controlled. In addition to the vibration cancellation, the electronic system produces the drive signal for the cryocooler and regulates the cooler temperature. The system employs a sinusoidal drive to reduce the amount of higher harmonic vibration. A digital signal processor (DSP) is used to perform the high speed vibration control. The Texas Instruments TMS320C31 processor is housed on a third-party board. A second board has analog-to-digital (A/D) and digital-to-analog (D/A) converters. The DSP was programmed in C.

The physical system consists of two sets of electronics. The first is housed in a case that is separate from the detector unit. It consists of the DSP boards and a custom board for power conditioning, some signal processing, and diagnostic LEDs. The other set of electronics includes an analog signal conditioning board and a digital pulse width modulated (PWM) amplifier board that drives the two voice coil actuators in the cryocooler.

The system achieves significant reduction in vibration on the fundamental frequency and several of its harmonics. RMS accleration reduction on the fundamental was $60 \mathrm{~dB}$. Overall acceleration levels were reduced from greater than $1 \mathrm{~g}$ to $1-10 \mathrm{mg}$.

Unfortunately, for the present report we were unable to include detailed information about the cryo-cooler performance. Delays in delivery of the cooler and subsequent delays in mounting the new system precluded an adequate determination of the device performance parameters. These will be determined and reported on at a later date.

\section{Introduction}

The vibration cancellation system was developed in support of the Lawrence Livermore National Laboratory (LLNL) project to produce a portable gamma ray detector. This sensor system is most effective when thermal noise is reduced by maintaining the detector at very low and constant temperature. The active cryocooler is an alternative to the conventional approach that employs a consumable reservoir of liquid Nitrogen to extract heat. It promises smaller mass and volume and hence greater portability. The chief difficulty with the active system is the introduction of detrimental vibration.

Vibration is a problem whether or not the detector is maintained at low temperature. Measurements are corrupted by motion of the detector. In a high vibration environment, any advantages of the active cooling compared to passive cooling are no longer relevant. The effort discussed in this report was conducted to develop a system that enabled 
operation with an active cooler. Thus, the main effort was directed towards reducing vibration.

Figure 1 shows the basic system components. The cryocooler extracts heat from the detector while under control of the cancellation system. The cancellation system uses no direct measurement from the detector. Instead, acceleration of the instrument body is minimized. Since the detector is mounted to this body, motion there is reduced as well.

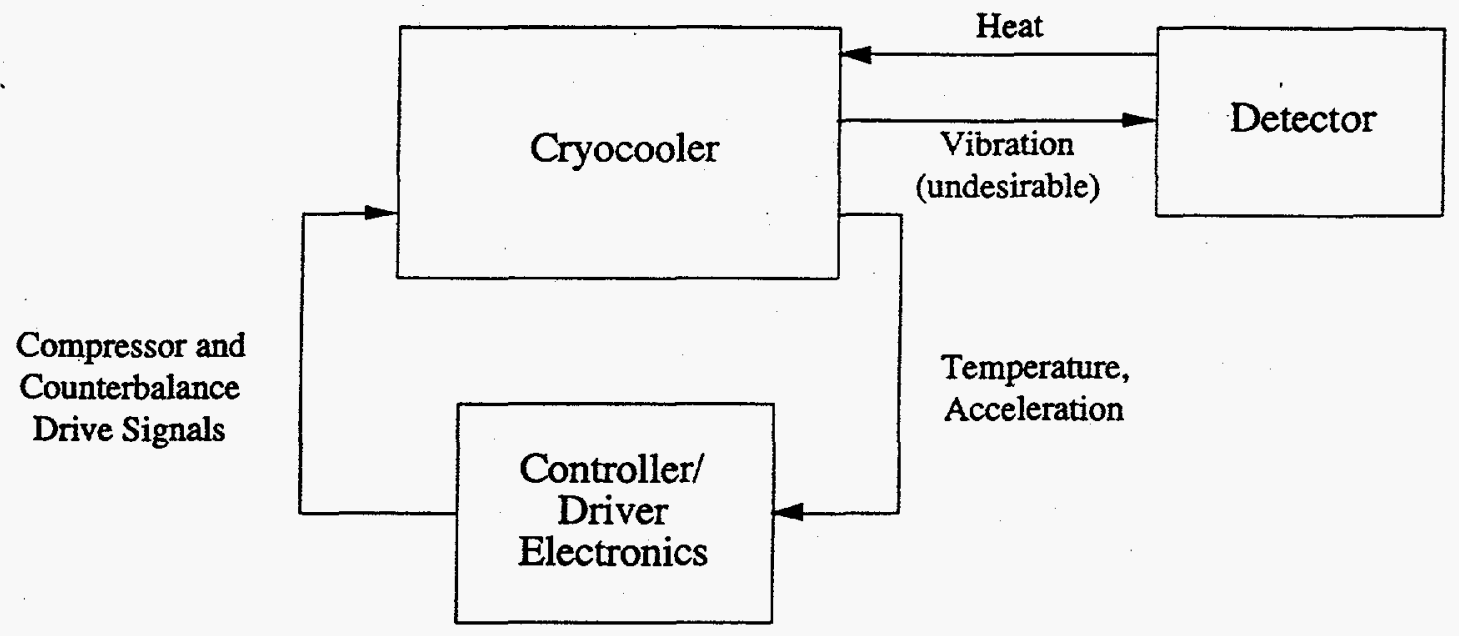

Figure 1: The control electronics were designed to allow active cooling without exposing the detector to detrimental vibration.

The remainder of this report describes the electronic vibration cancellation system. The system was developed prior to integration of the cryocooler with the gamma ray detector. In the next section, the electromechanical system is described and a simple model is introduced. Initial testing of the system in open and closed loop configurations is then summarized. The components that form the complete system are detailed next. These include the signal conditioning, digital signal processor (DSP) boards, DSP interface board, driver amplifier, software algorithm, and overall packaging. Results are summarized. Finally, suggestions for increased performance and further reduction in size of the system are provided. In the report, the compressor voice coil may be referred to as the "primary coil" and the counterbalance voice coil as the "secondary coil." The fundamental cooler drive frequency is designated $1 \mathrm{~F}$ and its harmonics $2 \mathrm{~F}$, $3 F$, etc.

\section{Initial Investigation of the System}

The cryocooler system investigated has two control inputs, the commands to the compressor and counterbalance actuators. The vibration suppression problem can be summarized as follows: command inputs to the compressor and the counterbalance to minimize the total acceleration of the cryocooler body while the cooler performs its required thermodynamic function.

\subsection{The Mechanical System}


All work was carried out without detailed knowledge of the proprietary mechanical design of the interior of the Sunpower cryocooler. The basic operation however was understood. A primary voice coil drives a compressor piston, doing thermodynamic work. A secondary (nominally identical) voice coil drives a counterbalance mass. The motion of this second mass was used to affect vibration cancellation. A smaller displacer mass also moves slightly out of phase with the compressor mass. From the standpoint of the counterbalance, the presence of the displacer and any other smaller components simply changes the magnitude and phase required for momentum cancellation.

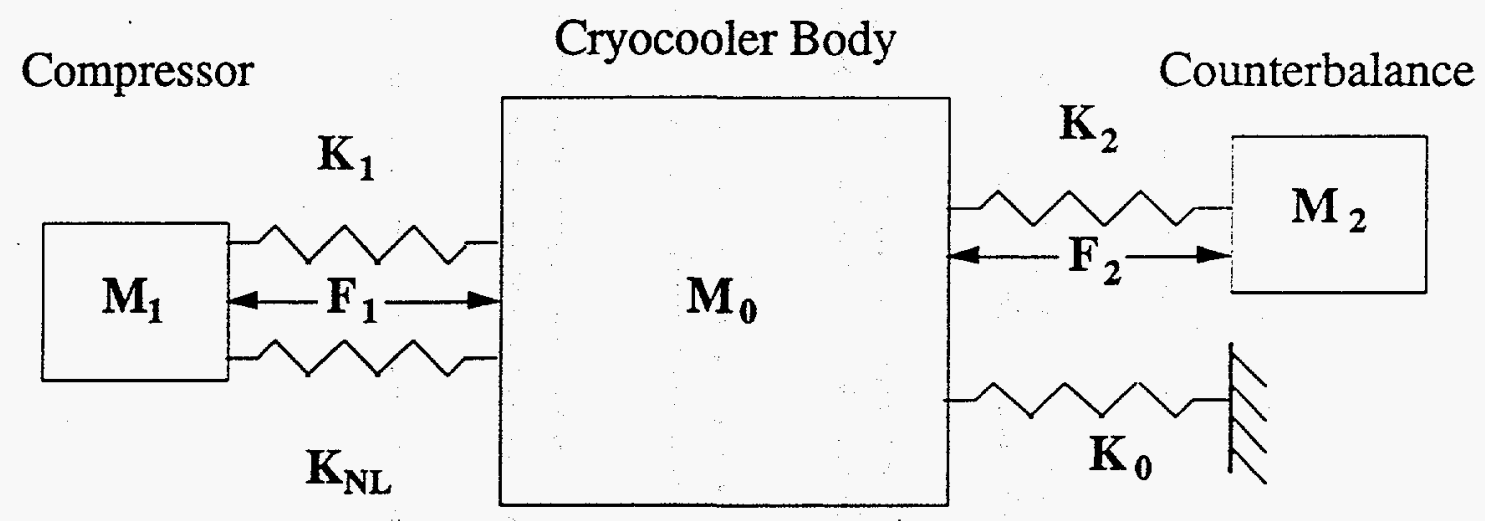

Figure 2: A simple mechanical model of the cryocooler system

A basic model of the system dynamics (Figure 2) was constructed. The model consists of three masses connected by springs. It does not include the displacer. The cryocooler body $\left(M_{0}\right)$ has at least 10 times more mass than the compressor $\left(M_{1}\right)$ and counterbalance $\left(M_{2}\right)$, which have nearly identical masses $\left(M_{1} \approx M_{2}\right)$. The spring $K_{0}$ connects the cooler body to ground. In the final system, this spring represents compliance of the mounts that isolate the cooler. The control approach used assumes that this spring is soft, such that the frequency of the mode associated with $\left(M_{0}\right)$ and $K_{0}$ is low (a few Hertz). In this case, modes of the base do not appear in measurements of the motion of the cooler body. This was the case in the tests conducted, in which the cooler sat on a 4 inch thick piece of foam. A 5-7 Hz rocking mode was introduced by the foam.

Even with passive isolation from base dynamics, the cooler itself possess other mechanical modes at frequencies well above those associated with the simple massspring system. Modes at 300 and $460 \mathrm{~Hz}$ were noted and later notched in the system that was delivered. Any mechanical redesign of the cooler is likely to change the frequencies on these internal modes.

With a soft spring $K_{0}$, the three-mass model adequately represents the system. The counterbalance spring $\left(K_{2}\right)$ is a simple linear one. The compressor spring $\left(K_{1}\right)$ is also linear. The nonlinear spring $\left(K_{\mathrm{NL}}\right)$ represents the effect of gas dynamics. This nonlinearity tends to introduce harmonics of any driving frequency in the response. The nonlinearity does decrease some as the temperature and pressure of the gas increase and motion is reduced. 


\subsection{Plant Transfer Function Model}

The expected transfer function from one of the voice coil actuators to the acceleration of the cooler body is shown in Figure 3. In this case, the nonlinear spring was ignored and the mass and stiffness of the compressor and counterbalance subsystems were identical. When those quantities are slightly different, two modes which are close in frequency are expected.

For the compressor, the designed drive frequency is just below the resonance. Some mechanical amplification (a factor of 2--5) is therefore used to increase efficiency. When the compressor is driven at a single frequency $1 \mathrm{~F}(58.65 \mathrm{~Hz}$ was used), the nonlinearity in the system results in acceleration response at harmonics of the fundamental drive frequency.

The same approximate transfer function applies for the counterbalance. The counterbalance must input energy at the frequencies of the higher harmonics to provide momentum cancellation. The $40 \mathrm{~dB} /$ decade rolloff above the resonance implies reduced authority at the higher harmonics. The authority at frequency $10 \mathrm{~F}$ is only about $1 \%$ of that for the fundamental (1F). This became important in controlling the amplitude of the output from the digital computer. That signal contained large components of the higher harmonics.

The plant phase at frequency $1 \mathrm{~F}$ is $0^{\circ}$ (or $180^{\circ}$ ), i.e., exactly in (or out of) phase. The phase for all higher harmonics is $180^{\circ}$ lagged from that phase. This is accounted for in the cancellation algorithm. 

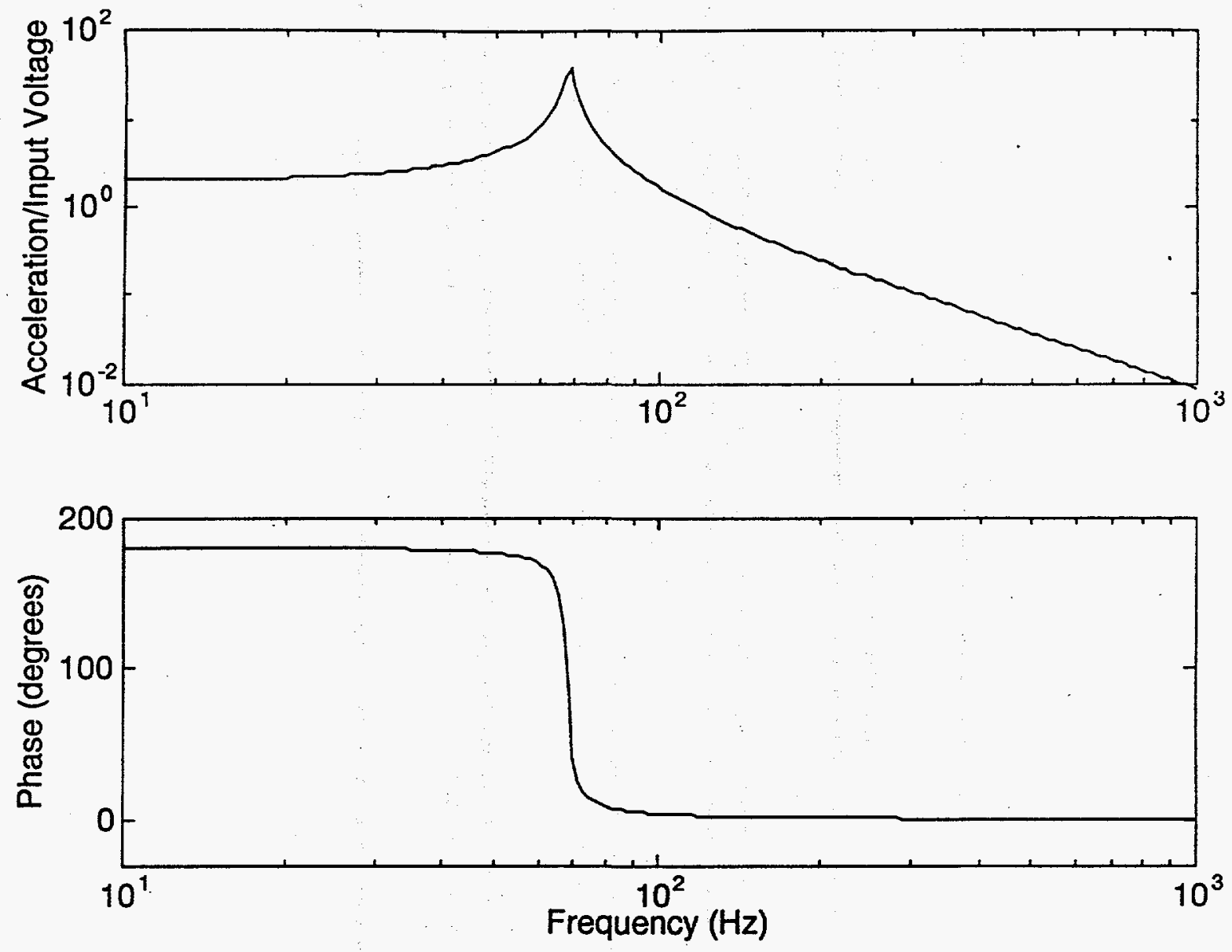

Figure 3: Expected transfer function between commanded voltage to compressor or counterbalance actuator and acceleration of cooler body

\subsection{Electromechanical Coupling}

As purely electrical systems, the two voice coil actuators are each well modeled as a series resistor $(R)$ and inductor $(L)$. The design values of $R=0.3 \sim \Omega$ and $L=6.3 \sim \mathrm{mH}$ were confirmed by measurement. With these values, the $R / L$ corner frequency is about $8 \mathrm{~Hz}$. Above this frequency, the inductor dominates the impedance.

Each of the voice coil actuators introduces electromechanical coupling into the system. Thus, when either of the two voice coils is shorted or left open circuited, the frequency of the modes changes, by as much as 10-15\%. The output impedance of the amplifier used to drive either coil, i.e., whether the amplifier commanded voltage or current, therefore influenced the system dynamics. Both current and voltage amplifiers were used at various stages of the effort. The final system employed voltage amplifiers. These tended to increase both the frequency and the damping of the massspring modes. A simple model of the system can be derived from the following two equations, which model a voice coil actuator driving a mass-spring system. The total voltage is given by 


$$
V=I R+L \frac{d I}{d t}+\beta \frac{d x}{d t},
$$

where $I, R, L$ are the current, resistance, and inductance, $\beta$ is the motor constant, and $x$ is the displacement of the mass, $m$. The third term represents the contribution of back EMF. The commanded force is proportional to both the current and the acceleration,

$$
F=m \frac{d x}{d t}=\beta I
$$

The transfer function from the secondary coil input to the body acceleration contains the mode corresponding to motion of the counterbalance mass in one direction and the body and compressor masses in the opposite direction. The frequency of this mode with current drive was about $61 \mathrm{~Hz}$, with a $Q$ of about 15 . With a voltage drive, the frequency was $70 \mathrm{~Hz}$, with a $Q$ of about 5 . Note that the frequency is not 58.65 $\mathrm{Hz}$. If the cryocooler body were clamped, the frequency would decrease further, towards $58.65 \mathrm{~Hz}$. The mode shape would consist only of the counterbalance mass bouncing on a rigid base.

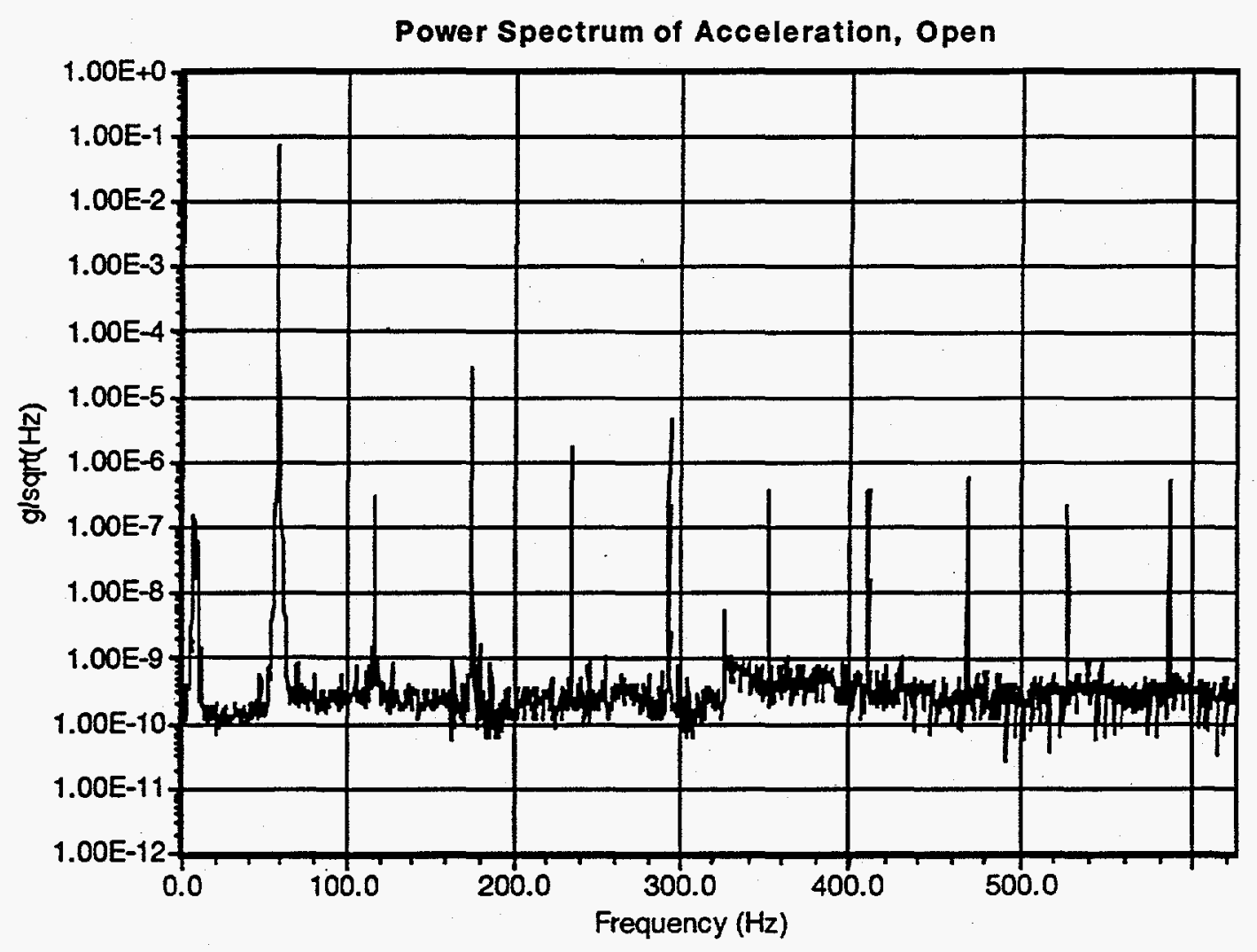

Figure 4: Measured acceleration spectrum in response to a $58.65 \mathrm{~Hz}$ sinusoidal input to the compressor coil 


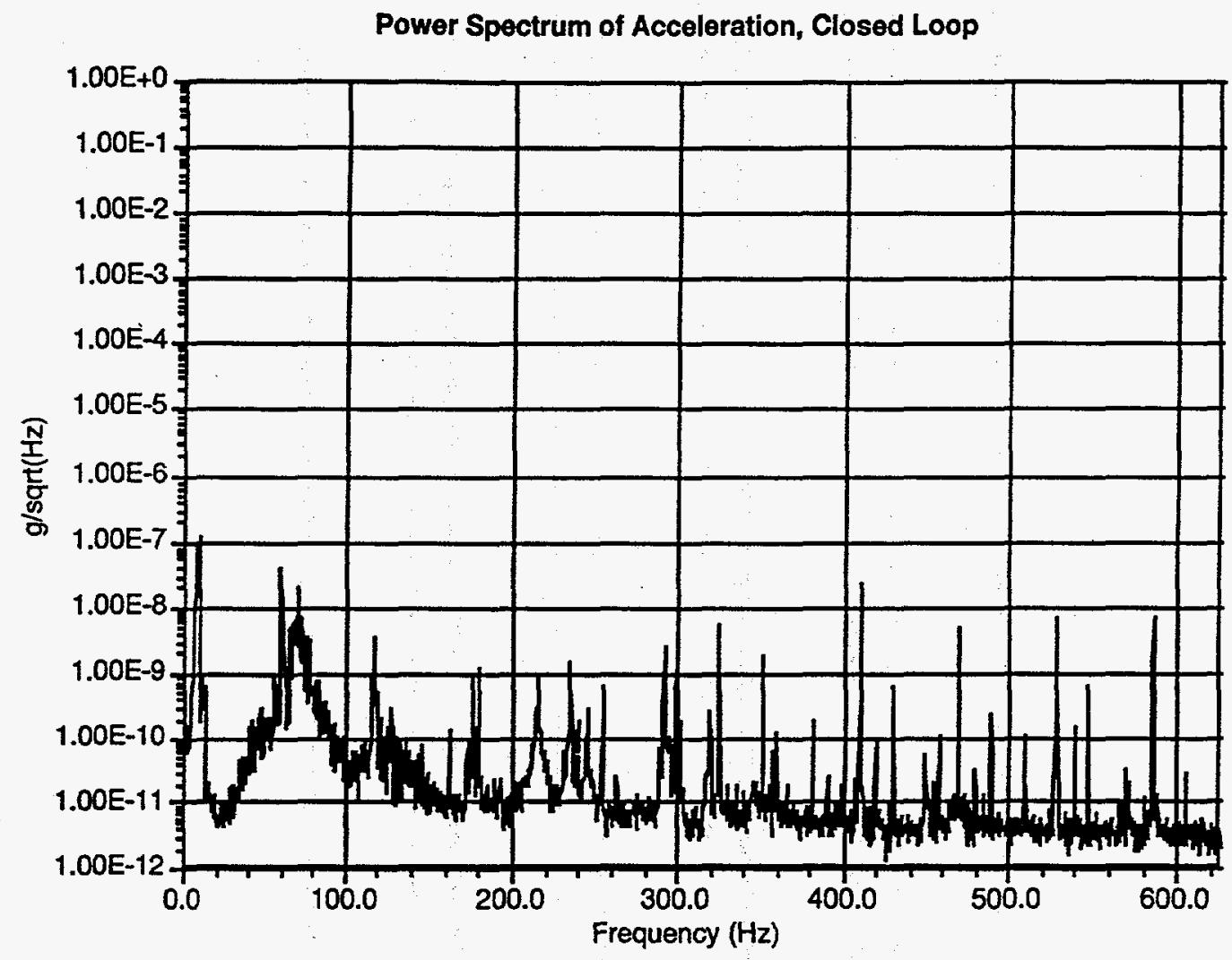

Figure 5: Measured acceleration spectrum in response due to a closed loop secondary coil active vibration control

\subsection{Nonlinear Response and Plant Transfer Function}

The body acceleration contains a significant amount of high frequency response. Of course, the displacement at high frequency is attenuated by $1 / \omega^{2}$. Figure 4 illustrates the acceleration in response to drive of the primary coil. There is measurable acceleration at each of the harmonic frequencies and that the accelerations are considerably lower than the fundamental frequency response. The same acceleration at two frequencies results in a quadratically larger displacement for the lower of those two frequencies.

Figure 6 shows the time domain version of the acceleration. Note the periodic appearance of the $3.1 \mathrm{kHz}$ signal each time the accelerometer reaches one end of its excursion. The source of this high frequency motion is unclear. Possibilities include internal valves, impact events, or local resonances. There is no significant audible component to the response. The main implication for the control work was that this signal needed to be attenuated. The only real way to eliminate this type of response is by redesign of internal components. Note again that although the acceleration time signal is choppy, the twice-integrated displacement is extremely smooth. 


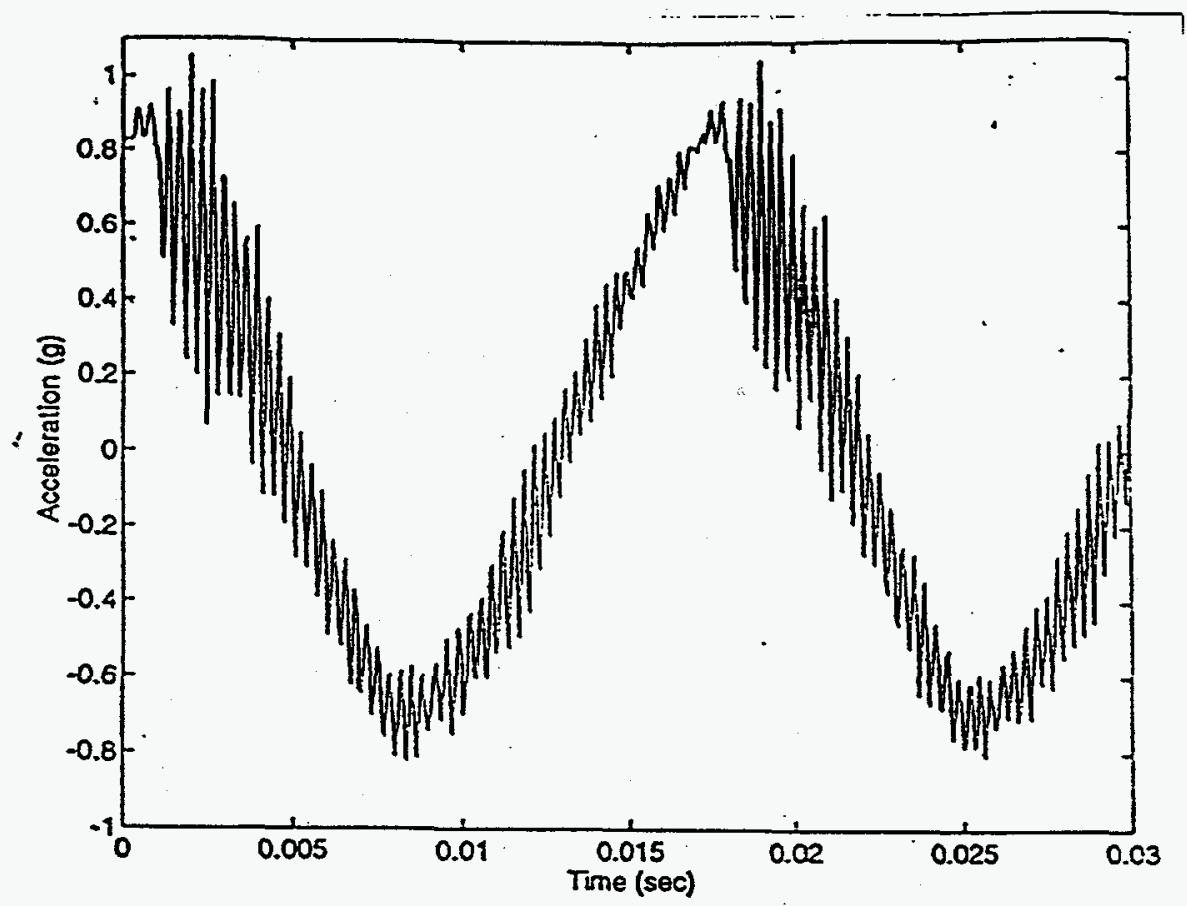

Figure 6: Measured acceleration time responce due to a $58.65 \mathrm{~Hz}$ sinusoidal input to the compressor coil. 

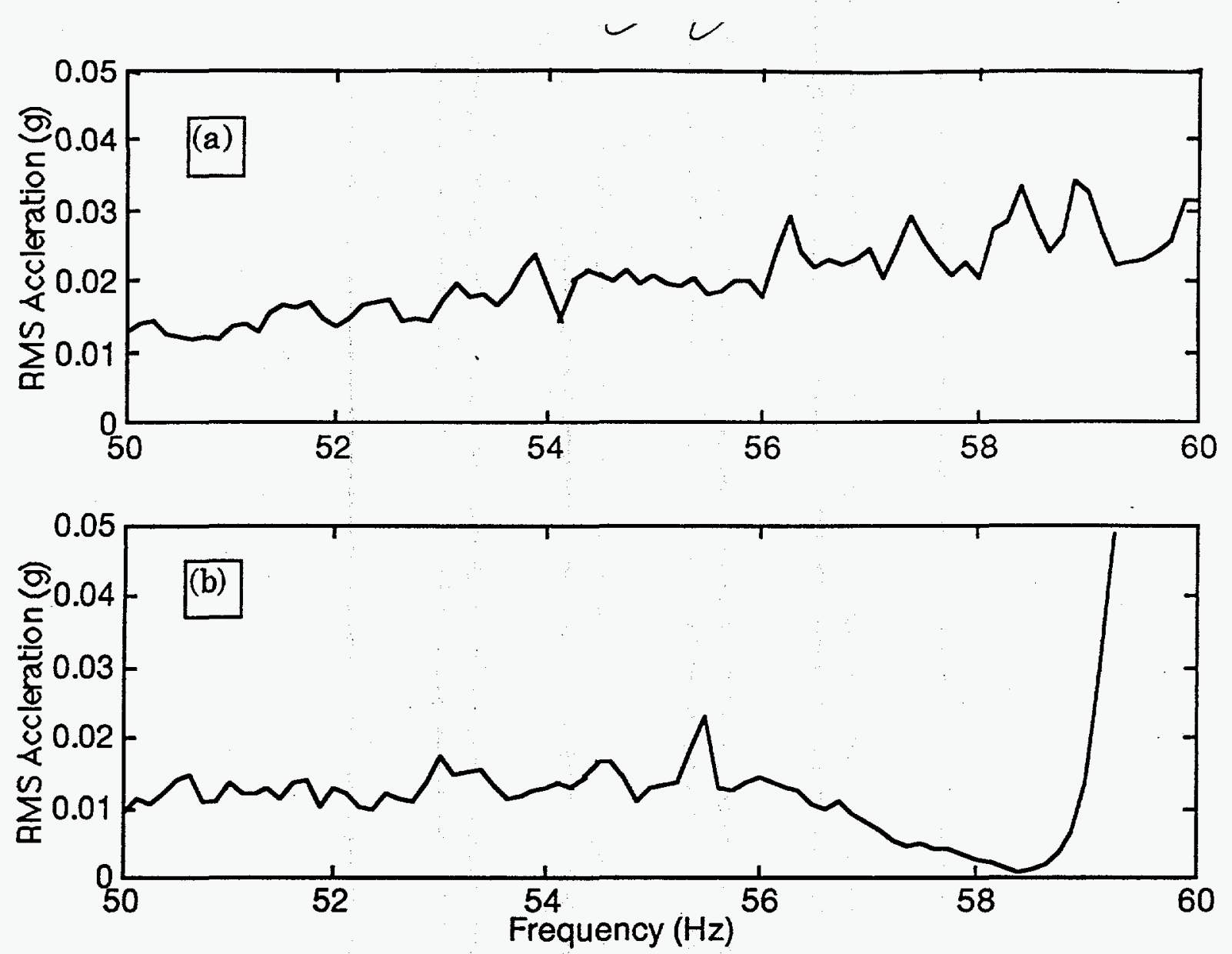

Figure 7: Reduction in acceleration when the counterbalance acts as a passive absorber (a) closed circuit (b) open circuit.

\subsection{Passive Absorber Effectiveness}

A series of tests was done to measure the effectiveness of the counterbalance as a passive vibration absorber. Initially, it was hoped that substantial reduction in body acceleration could be achieved in this way. Figure 7 indicates a typical result. The compressor coil was driven with a current amplifier at various frequencies near the operating frequency of $58.65 \mathrm{~Hz}$. With the counterbalance coil short-circuited, it contributed nothing to reduction in acceleration. With the coil open-circuited, and therefore free to generate a back EMF, there was reduction near the resonance of the counterbalance. The greatest improvement was only a factor of about 2.5. The counterbalance could be made more effective in this passive mode by mechanical redesign and increasing of the mechanical $Q$ by removing lossy components in the load path.

\subsection{Initial Control Experiments}

The first tests targeted reduction of the response at $1 \mathrm{~F}$. This was first attempted by a crude open loop exploration of the proper magnitude and phase for cancellation. One result of these tests, which achieved $40 \mathrm{~dB}$ rms reduction, was the indication that the 
required input signal was not exactly out-of-phase with the compressor command. A substantial phase difference, dependent on the temperature of the cooler, was noted. In the first closed loop control attempted, the response of the fundamental was reduced by $50 \mathrm{~dB}$ using a classical feedback compensator.

In this case, which used a current amplifier, the presence of a lightly damped mode near $58.65 \mathrm{~Hz}$ (at $61 \mathrm{~Hz}$ ) was deemed unacceptable. A voltage mode amplifier was therefore used. Still, the response of the $70 \mathrm{~Hz}$ mode had to be attenuated by a notch in the compensator. Subsequently, a voltage mode amplifier was used for both coils. The notching of the mass-spring mode was incorporated in the final system to allow high gain (more than $60 \mathrm{~dB}$ ) while maintaining stability robustness.

\section{Sensor Signal Conditioning}

The analog signal conditioning board includes precision circuits for a cryogenic temperature sensor and an accelerometer. Conditioning for the temperature sensor was designed for a Lake Shore DT-470 temperature diode. Figure 8 shows the diode forward voltage drop as a function of temperature. The temperature range of interest ( $70 \mathrm{~K}$ to $300+\mathrm{K}$ ) maintains the diode in the nearly linear range.

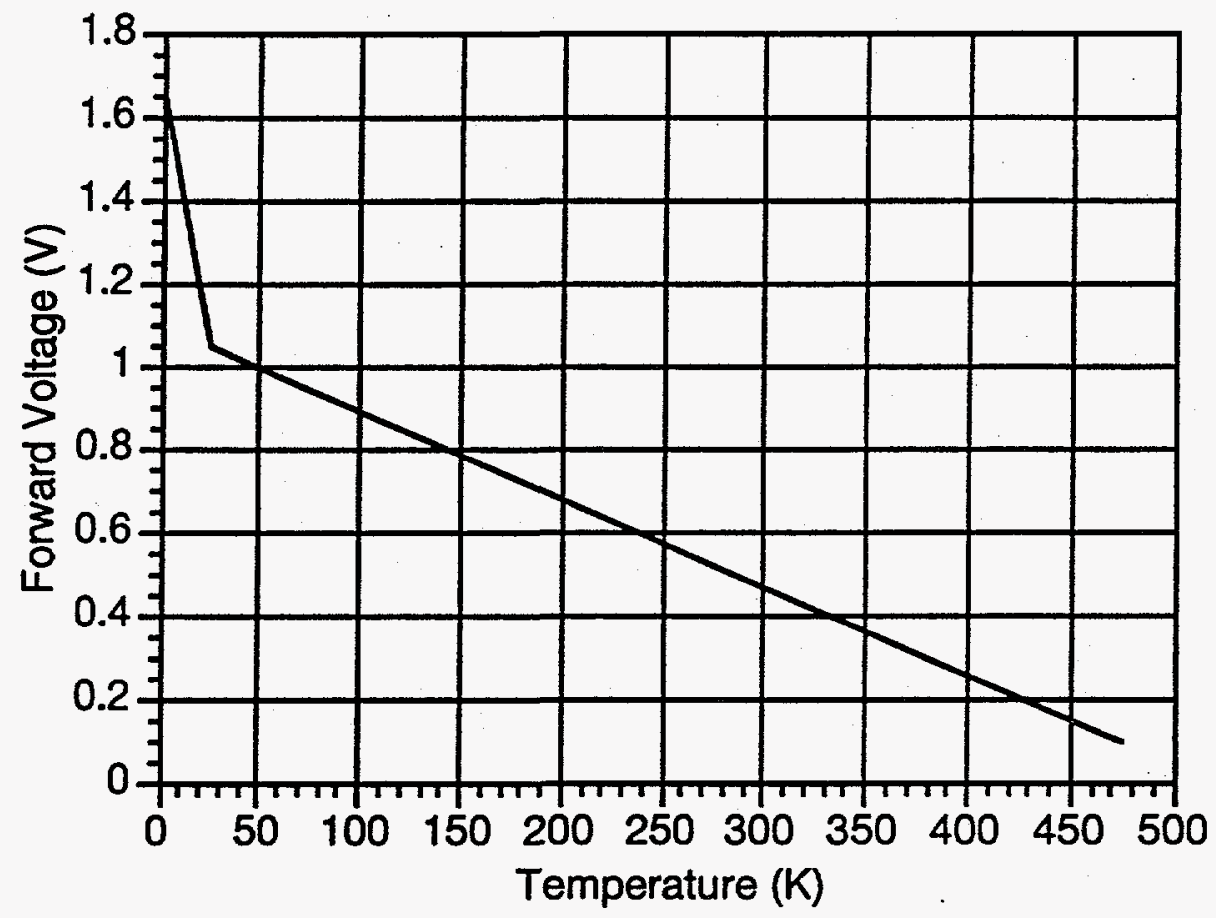

Figure 8: Forward voltage drop of the Lake Shore DT-470 temperature diode (Lake Shore Cryonics Standard Curve \#10)

Acceleration signal conditioning was designed for one or two inputs from highimpedance Endevco 7703-50 accelerometers $(50 \mathrm{pC} / \mathrm{g})$. The acceleration output has an optional low-pass filter that is switchable by jumper. During operation the board resides next to a switching amplifier. A copper housing protects against electromagnetic interference from the switcher. 
$\checkmark v-c$

The temperature circuit outputs a DC signal that nominally ranges from 0.5 to 4.5 Volts for temperatures from $77 \mathrm{~K}$ to $300 \mathrm{~K}$. The Operational Amplifier (Opamp) labeled OT01 provides a constant current of $10 \mu \mathrm{A}$ to the diode and the resulting voltage drop indicates temperature. A board-mounted voltage reference provides a precise voltage to maintain the constant current. Voltage across the diode is differenced with an amplifier. High input impedances are provided with a three amplifier design, since the diode's source impedance is commonly greater than $100 \mathrm{KOhms}$. Layout of the differencing amplifier provides high common-mode rejection, and high input impedance with a relatively simple, compact design. Its output is offset before, and amplified at, OT05. Operational amplifier trims are unused for maximum common mode rejection. Auxiliary resistor locations in the feedback of OT05 and in the offset circuit enable fine tuning on both.

The output of the temperature conditioning for a specific unit may be slightly different from the nominal $0.558 \mathrm{~V} / \mathrm{K}$ and $77 \mathrm{~K}$ voltage of $0.5 \mathrm{~V}$. Part of any small discrepancy may be introduced in additional conditioning (a nominal unity-gain inverter on the unit delivered) on the board housed in the battery enclosure. These values can be adjusted in software for a specific unit if greater accuracy is needed. Note that the software variable $K p e r A D C$ is 10 times the analog circuit $V / K$ value.

Acceleration conditioning provides a calibrated output from one or two accelerometers. If connected, the outputs from two accelerometers are summed and the effective gain is doubled. The positive leg of amplifier OA01 is bootstrapped to make circuitry both simple and compact. Capacitance of the CA01 must be around 4 times that of the accelerometer and cable to minimize overshoot and/or ringing. The positive input to OA01 is jumped to avoid degradation from the low insulation resistance of traces on the board. A 4-pole low-pass Butterworth filter offers optional filtering starting at 1000 $\mathrm{Hz}$.

Opamps were selected carefully to serve each application. The temperature conditioner uses OP37 or equivalent amplifiers. They provide low noise, low voltage offsets, and high common mode rejection. The acceleration conditioner uses an OP41 for low current bias and noise.

The copper housing protects against electromagnetic interference. Holes were minimized since they deteriorate performance substantially. The terminal block connectors on the analog board serve this purpose. With this design all conductors may remain in place during removal of the box if excess wire is sealed inside. The two existing holes should be sealed with conductive epoxy after final mounting.

\section{Power System}

\subsection{System for Initial Testing}

A Copley Controls Model 306-1 switching amplifier (10 A peak) was used to drive the primary (compressor) voice coil. A Kepco BP36-6M (40 V, 6 A peak) linear amplifier, in voltage mode, was first used to drive the counterbalance actuator. The Copley amp was mounted to a 48 VDC power supply. The Kepco amp was plugged into the wall. Based on these tests, we expect to meet the 9 Watt power budget for the cancellation system. The primary drive system will also be highly efficient. The main consumer of power is the drive for the compressor. The power required by the counterbalance actuator is reduced in the closed loop case. 


\subsection{Custom System}

The power driver for the cyro-cooler is a modified version of a circuit developed by AeroAstro for driving satellite magnetic torque coils. This circuit contains three sections: a power MOSFET H-bridge circuit, FET gate drive circuits and a digital pulse width modulator.

The first generation circuit of this type has been in flight operation on the ALEXIS small satellite for over one year with no problems of any sort. The second generation version has been developed for the HETE SC and is considerably more compact and integrated. This design is currently undergoing environmental testing and system integration. Design, materials and workmanship of these devices is suited for space flight operation, including vacuum operation and radiation tolerance. Several modifications are required for driving the cyro-cooler coils:

1. Higher currents. The cyro-cooler has $0.03 \mathrm{ohms} D C$ resistance and $0.3 \mathrm{ohms}$ impedance at the operating frequency of $60 \mathrm{~Hz}$, this is a considerable increase in current. We changed the drive transistors to types exhibiting lower on-resistance (Rds) and in packages with higher power dissipation capability.

2. EMI suppression. The higher current draw will result in larger EMI, which might adversely effect detector operation. We reduced this effect by the addition of hash chokes to the power input section.

3. Higher switching rates. Good control of higher harmonic vibration modes $(600$ $\mathrm{Hz}$ ) will require a higher switching rate than the nominal $4 \mathrm{kHz}$. We operated the PWM section at $20 \mathrm{kHz}$ switching rate, the only modification needed is a different clock rate $(20-40 \mathrm{Mhz})$ to the control chip.

4. Digital interface. The current design uses a proprietary serial interface, we designed the new control chip to accept parallel digital data from the control computer (TI DSP C31) but a simple synchronous serial interface is also possible (Motorola HC11).

5. Physical layout. We built a pair of drivers onto a single four-by-four inch board and to heat sink the power transistors directly to the housing.

Specifications for the drivers are as follows: two H-bridge drivers with digital PWM control $10 \mathrm{kHz}$ switching rate 11 bit plus sign resolution digital control interface operating voltage $18 \mathrm{~V}-32 \mathrm{~V}, 6 \mathrm{~A}$ continuous drive capability per channel.

The digital PWM chip was redesigned to overcome several initial difficulties. These included occasional glitches and problems at zero crossings. The latter problem was addressed by not allowing the chip to command the values $-1,0$, or +1 (The allowable values now range from -2046 to +2046 ). The driver board was designed to operate with a $10 \mathrm{MHz}$ clock, and therefore a $9.765 \mathrm{kHz}(20 \mathrm{MHz} / 2048)$ output rate. The system may be able to run at significantly higher rates, a feature which substantially reduces audible noise. The driver board clock can be easily changed. The ultimate system would run synchronously, with the DSP board and the driver board running at the same rate. This capability is available in the latest generation digital PWM chip, but is not 
$v-v$

currently exploited. Implementation would require minor modifications to both hardware and software.

\section{Prototype Digital Control System}

The vibration cancellation digital control system was investigated prior to availability of the digital PWM driver board. This development is summarized here. The main distinction from the final system is the use of a different cancellation algorithm.

\subsection{General}

The prototype digital control system was implemented on a Texas Instruments TMS320C31 DSP hosted on board. The control system has two inputs and two outputs and fulfills two functions

1. It generates the primary signal driving the compressor, principally a sinusoid at $1 F=58.65 \mathrm{~Hz}$. The amplitude of the signal is regulated depending on the temperature to ensure a fast and safe cooling transient

2. Based on an accelerometer error signal, it adaptively generates the secondary signal driving the counterbalance. The control signal is generated by a filtered-X LMS algorithm and contains the first 10 tones of the disturbance.

\subsection{Internal Signal Generation}

The control algorithm requires an internal signal generator to produce tones at $1 \mathrm{~F}$, 2F, ..., 10F. A cosine look-up table with 100 points was used as a signal generator. The sampling frequency range to 100 times $1 F=5.865 \mathrm{kHz}$. Using pointers and modulo 100 arithmetic, $\mathrm{nF}$ tones, where $\mathbf{n}$ is an integer, were generated by reading every other $\mathbf{n}$ elements of the cosine look-up table and wrapping around when the index had exceeded the size of the table. To generate a sine, or to shift the phase of the cosine, it is only necessary to shift the index in the look-up table, still with modulo 100 arithmetic. This approach proved computationally very efficient.

\subsection{Primary Driving Signal}

The primary signal is a pure tone at $1 \mathrm{~F}$. The amplitude of the primary signal was constant in this implementation of the control system. The work was carried out at a temperature of $100 \mathrm{~K}$. In a separate series of tests, preshaping the input to the nonlinear system was attempted. A $2 F$ tone was added in quadrature with an amplitude equal to $2 \%$ of the first tone. Adding the 2F tone in the primary signal driving the compressor was shown to reduce the higher harmonic content of the disturbance. This approach shows promise for attacking perhaps half of the amplitude of vibration at each of the harmonics. More substantial reductions may be possible, and could be maximized by gain scheduling the preshaped input with temperature and/or amplitude.

\subsection{The Filtered-X LMS Algorithm}


The implementation of the filtered-X LMS algorithm consisted of 10 filters running in parallel, each utilizing a pair of 2 point FIR filters to generate the adaptation and the output signal. The $1 \mathrm{~F}$ to $10 \mathrm{~F}$ LMS external reference signals were generated internally since the control system drove both the compressor and the counterbalance mass. For the $n F$ harmonic, an integer index $I_{n}(\bmod 100)$ indicated the current phase and was updated at every step by $n$. In order to have a better accuracy on the phase, the 2 points are selected roughly $1 / 4$ period apart. The delay was converted into an integer shift $D_{n}$ closest to $100 / n / 4$ (e.g. D1 $=25, \mathrm{D} 2=12$, etc.). The $n \mathrm{~F}$ part of the output signal is, therefore:

$$
y\left(I_{n}\right)=\omega 1_{n} \cos \left(I_{n}\right)+\omega 0_{n} * \cos \left(I_{n}-D_{n}\right),
$$

where $\omega 1_{n}$ and $\omega 0_{n}$ are the $n \mathrm{~F}$ LMS weights and where $\cos (I)$ is the Ith element of the look-up table. The weights of each tone were updated every $D_{n}$ steps, that is, roughly 4 times per tonal cycle, at $234 \mathrm{~Hz}$ for $1 \mathrm{~F}, 488.75 \mathrm{~Hz}$ for $2 \mathrm{~F}\left(D_{2}=12\right), \ldots, 2.9 \mathrm{kHz}$ for $10 \mathrm{~F}$ $\left(D_{10}=2\right)$ The accelerometer signal, sampled at $5865 \mathrm{~Hz}$, was averaged over quarter cycles to update the weights of each tone. The error $E_{n}$, used in the $n F$ filter, averaged $D_{n}$ sampled accelerometer readings.

The LMS algorithm requires the knowledge of the plant transfer function at the disturbance frequencies (1F to $10 \mathrm{~F}$ ). Because the disturbance frequency was computer driven and extremely stable, the transfer function was measured at $58.65 \mathrm{~Hz}, 117.3 \mathrm{~Hz}$, ..., $586.5 \mathrm{~Hz}$ using a computer driven sine wave excitation. An external 8-pole, lowpass filter at $1 \mathrm{kHz}$ was later added for better anti-aliasing on the accelerometer signal. The filter was independently identified and the measured gains and phases at $1 \mathrm{~F}$ to 10F were incorporated to the measured plant gains and phases. The 45 degree phase lag introduced by the averaging was not taken into account. The gain $A_{n}$ and phase $F_{n}$ of the resulting plant transfer function are later transformed into a 2-point FIR filter. The filter weights $g 1$ and $g 0$ are calculated so that:

$$
A_{n} \cos \left(\omega_{n} t_{s}+F_{n}\right)=g 1_{n} \cos \left(I_{n}\right)+g 0_{n} \cos \left(I_{n}-D_{n}\right)
$$

where $t_{s}$ is the sample time corresponding to the index $I_{n}$.

The adaptation was implemented with no loss factor, meaning a theoretically infinite loop gain at $1 F$ to $10 \mathrm{~F}$. The loop transfer functions were not measured.

\section{5 dSPACE 1102}

The dSPACE 1102 board was used for all of the development work. The strenght of the dSPACE system is its ease of use including simplicity in programming. Initially, the two 16-bit A/D converters were used to implement the control. A switch to 12-bit A/D's was made to reduce the time required per sample period. There was no noticeable sacrifice in performance. Twelve-bit $A / D$ 's and $D / A$ 's were used in subsequent development. The dSPACE libraries were adapted for use with the target DSP system. This enabled running with either system with limited changes in the C code. An important effect of this implementation is that all variables within the software are referenced to $\mathrm{a}-1$ to +1 value, corresponding to $-10 \mathrm{~V}$ and $+10 \mathrm{~V}$ at the 
A/D's. One limitation of the dSPACE system is the digital I/O speed. The output is particularly important for driving the digital PWM amplifiers. The TI's C14 component used was not adequate to perform the three writes per cycle and still allow time for $\mathrm{A} / \mathrm{D}$ reads and other computation. The possibility of a workaround was discussed with dSPACE officials. This was not pursued in depth, but could be revisited later if necessary.

\section{Software and Algorithm}

The present document describes the cryocooler control software as implemented at the end of September 1994. The software is currently written to operate with the dSpace real-time development system which uses a TMS320C31 DSP hosted on dSPACE board in a PC.

\subsection{Control Software Functionality}

The cryocooler control software performs three main functions

Temperature Regulation. this task consists of generating the proper compressor drive signal based on temperature measurements. Its goal is to reduce the cold finger temperature as fast as possible and maintain it at $77 \mathrm{~K}$

Vibration Suppression. this task consists of generating the proper balancer drive signal based on accelerometer measurements. Its goal is to minimize the first 10 harmonics of the vibration generated by the compressor at the drive frequency.

Safeguard. this task consists of taking the proper actions to guarantee the mechanical and electrical integrity of the system, with possible shut-down of the system to prevent any damage of the compressor, the balancer and most importantly the main sensor.

\subsection{Control Realization}

The control software is implemented digitally on a single TMS320C31. The Compressor Drive Signal (CDS) and the Balancer Drive Signal (BDS) are generated digitally by the controller. The base sampling and computation cycle rate is $F S=5,865$ $\mathrm{Hz}$, giving a 100 point resolution for the fundamental harmonic FO $=58.65 \mathrm{~Hz}, 10$ point resolution for the 10th harmonic. The three main functions are constituted of several subfunctions which are not synchronized and run at different rates. The following outlines the sub-functions.

\subsubsection{Temperature Regulation}

Temperature Signal Conditioning, run at base sampling rate $\mathrm{FS}=5,865 \mathrm{~Hz}$,

Temperature Control Logic, run at FT $=0.98 \mathrm{~Hz}=\mathrm{FS} / 6,000$.

CDS Generation, run at base sampling rate FS 


\subsubsection{Vibration Suppression}

Accelerometer Signal Conditioning run at base sampling rate FS.

Sequential Adaptation Logic. The logic updates one harmonic during 4 harmonic cycles at base sampling rate FS. After 4 harmonic cycles, the next harmonic is updated, until all 10 have been updated once, then cycles through again. Complete update cycles are run at $5.12 \mathrm{~Hz}$ (Complete Update Cycle period $=4$ ( ( $17 \mathrm{~ms}+17 / 2$ $\mathrm{ms}+17 / 3 \mathrm{~ms}+\ldots+17 / 10 \mathrm{~ms})=190 \mathrm{~ms})$.

BDS Generation run at base sampling rate FS.

\subsubsection{Safeguard}

CDS Amplitude Limiter Logic, run at FT.

CDS Limiter run at base sampling rate, FS.

BDS Interrupt run at base sampling rate FS.

\subsection{Function Details}

\subsubsection{Temperature Regulation}

The voltage read by the A/D converter is nominally related to the temperature by $\mathrm{T}=27.88 \mathrm{~V}+77$, where the temperature, $\mathrm{T}$, is in Kelvins. The temperature is sampled at FS and is accumulated over NTemp $=6000$ samples in the variable Temp. After 6000 counts, the temperature logic is executed once and the A/D temperature channel is not read.

The temperature control logic consists of a Proportional plus Integral (PI) control loop running at FT and an Amplitude Limit Ramp-Up module: The PI network uses an anti-wind-up feature on the integrator to prevent excessive error to accumulate. The integral of the temperature error, STemp, is limited to 1.5 $V_{c} M a x / K I$, where $V_{c} M a x$ is a voltage limit on the compressor drive voltage andKI is the gain on the integral term. The gain of the PI loops are selected to give the closed loop system a 1 minute period and critical damping. These are adjustable in the software.

The Amplitude Limit Ramp-Up is an open-loop integrator running at FT. The input to the integrator is VAsct $=0.04$ Volt / Temperature Logic Update Cycle (the Ramp-Up input can be either turned off, or can be set to VDsct $=-0.1$ Volt / Temperature Logic Update Cycle, by the Safeguard Function.) The output of the Ramp-Up module is $V_{c}$, or maximum allowed CDS signal amplitude. $V_{c}$ is set to zero when the system is started. With the currently selected value (which depends on the current amplifier used to drive the compressor), it takes about 60 seconds for $V_{c}$ to reach a value corresponding to maximum safe cooling down conditions. The thermodynamic work performed by the compressor increases as the cold finger temperature diminishes. A given command voltage to the compressor 
produces a larger motion when the cold finger is hot than when the cold finger is cold. The Temperature Control Logic is not concerned, therefore, with reducing the CDS Amplitude Limit; the Safeguard function fulfills this task.

The generation of the compressor input is fairly straightforward. The CDS is the product of a Base Signal generated by a internal signal generator times the amplitude generated by the Temperature Control Logic. The Base Signal is produced at FS using a look-up table Tcos and pointers.

\subsubsection{Vibration Suppression}

Signal Conditioning: The accelerometer signal sampled at FS is passed through a digital notch filter also operating at FS. The notch filter realizes the following continuous transfer function using pole/zero mapping technique:

$$
H(s)=s^{2}+\frac{\Omega^{2}}{\left(s^{2}+\frac{\Omega}{Q} s+\Omega^{2}\right)}, \Omega / 2 \pi=68 h z, Q=10 .
$$

The notch filter allows one to use a higher gain in the LMS loop at $58.65 \mathrm{~Hz}$ and $117.3 \mathrm{~Hz}$ without destabilizing the fundamental balancer mode around $68 \mathrm{~Hz}$.

notches: $68,177,301,455 \mathrm{~Hz}$.

Sequential Adaptation Logic: The Sequential Adaptation Logic is run at FS and updates the weights used to generate each harmonic constituting the BDS. The logic adapts the weights of one harmonic at a time. The updating period for one harmonic covers 4 harmonic cycles. At the end of the 4 cycles, the logic switches to updating the weights of the next harmonic, and cycles through when all harmonics have been updated. The update algorithm is a regular filtered-X LMS algorithm. For $i$-th harmonic, the LMS algorithm uses a two weight FIR model of the plant and a delay line covering $N_{i}$ samples, $N_{i}$ being selected such that the delay $N_{i} / F S$ is as close as possible to a quarter period of ith harmonic. The FIR filter weights the current and the oldest sample of the delay line. With a fundamental period $1 / 58.65=17 \mathrm{~ms}$, the total update cycle takes $4((17+17 / 2+17 / 3$ $+\ldots+17 / 10) \mathrm{ms}=190 \mathrm{~ms}$. Complete update cycles thus occur at a rate of $5.12 \mathrm{~Hz}$. BDS Generation The BDS is generated at a rate of FS. It uses a single look-up table and pointers to produce the sum of ten harmonics form $58.65 \mathrm{~Hz}$ to $586.5 \mathrm{~Hz}$. The $i$ th BDS harmonic is the superposition of two signals, one cosine and one phase shifted cosine. The phase shift is realized using the same time-delay of $\mathrm{N}_{i}$ used in the LMS algorithm and provides a phase shift equal or close to $90 \mathrm{deg}$. Each signal is multiplied by the proper weight updated by the Sequential Adaptation Logic and accumulated before it is sent to the D/A.

\subsubsection{Safeguard}

CDS Amplitude Limiter Logic: The Amplitude Limit Ramp-Up run at FT receives two commands from the Safeguard function: one to maintain the Amplitude limit $\mathrm{V}_{\mathrm{c}}$ at its current value, the second to reduce the limit at $\mathrm{VD}_{\mathrm{sct}}=0.1 \mathrm{Volt} /$ 
Temperature Logic Update Cycle (-0.1 Volts/second approximately, total shut down in about 15 seconds). The Amplitude Limiter Safeguard Logic considers safe mechanical operating conditions and prevents the compressor from hitting mechanical stops and generating vibration levels harmful to the main sensor. The mechanical motion of the compressor is effectively indicated by the fundamental harmonic of the BDS. The Adaptation Logic does indeed ensure that the momentum delivered by the balancer equals that produced by the compressor. In preliminary open-loop tests, one can calibrate the safe operating limit indicated by the BDS. The compressor is run first open-loop (no vibration isolation) with the balancer stage shorted to reproduce the dynamics of the system driven by a voltage amplifier. The compressor is driven with increasing amplitudes until it starts hitting hard stops. The compressor is then turned off, its terminals short circuited, and the balancer is driven to reproduce the RMS acceleration recorded when the compressor started hitting. The BDS peak drive voltage is found to be 3.9 V. A safe operating limit was chosen at 3.6 V. Amplitude Limit is allowed to ramp-up in the Temperature Control Logic at rate $V A_{\text {sec }}$ until the BDS fundamental harmonic is above the safe operating limit. A second limit, the never-to-exceed limit, is set at 3.7 V. Crossing this limit triggers the input to the Amplitude Limit Ramp-Up to switch a large negative value for a quick reduction of the compressor amplitude $\left(\mathrm{VD}_{\mathrm{gct}}=0.1 \mathrm{~V} / \mathrm{Temperature} \mathrm{Logic} \mathrm{Update} \mathrm{Cycle.)}\right.$ CDS Limiter A voltage limit $V_{c} M a x$ is set on the CDS to guarantee electrical integrity in the driving amplifier. This limit is not very effective with a current amplifier driving the compressor since it is must be set very low at high temperature and low thermodynamic work, but then prevent the compressor to work hard enough when the temperature goes down. This limit will be effective with a voltage amplifier driving the compressor. BDS Interrupt To prevent instabilities in the LMS loop, the computed BDS is compared to a maximum voltage limit $V_{b} M a x$. The balancer drive signal is set to zero in case the threshold is crossed. $V_{b} M a x$ is currently set at 5.0 Volts. While the balancer is interrupted (zero voltage), the Adaptation Logic runs open loop. Since it has infinite gain at the disturbance harmonics, running open loop will further increase the computed BDS, reinforcing the violation of the BDS Interrupt limit. This will therefore effectively shut down the balancer completely.

\subsection{Failure Accommodation}

Several failure cases can be handled gracefully by the software in its current configuration: Vibration Suppression Loop Instability: The computed BDS is unstable and exceeds $V_{b} M a x$. The BDS Interrupt sets the BDS to zero whenever the computed BDS exceeds $V_{b} M a x$. This effectively turns off the balancer. Because the LMS is unstable, or runs open-loop as the balancer is turned off, the computed BDS never diminishes, guaranteeing a complete balancer shut-down. The fundamental harmonic of the computed BDS, growing unstably or open-loop, and remaining high, always exceeds the never-to-exceed limit. The Safeguard Logic interprets this as the compressor being run too hard and command the amplitude limit to decrease until the compressor is effectively shut-down. Balancer failure: The balancer being failed, the Vibration Suppression runs open-loop. Since it has infinite loop gain at the disturbance frequency, the computed BDS grows endlessly. The BDS fundamental starts exceeding the never-to-exceed limit and remains high. The safeguard interprets 
the BDS being high as the compressor being run to hard. It commands the CDS amplitude limit to decrease until the compressor is effectively shut-down. Compressor failure (resulting in high vibration levels): The Vibration Suppression loop increases the BDS to compensate for the larger disturbance due to the compressor. The BDS starts exceeding the BDS Interrupt limit $V_{b} M a x$. The BDS Interrupt starts to shut down the balancer. The Vibration Suppression loop is being opened and the computed BDS grows endlessly, thus reinforcing the shut-down of the balancer. The computed BDS fundamental is high because of the failure or because the balancer is being shut down. It starts exceeding the never-to-exceed limit and remains high. The Safeguard Logic commands a reduction of the CDS Amplitude Limit until the compressor is effectively shut down.

\subsubsection{Remaining Work}

Most of the work remaining concerns the Safeguard function. Most likely failures are well covered by the current software. The following modifications should however be made: After effective shut-down of the balancer, the computed BDS is run open-loop. As it reaches a specified threshold, an error should be declared to effectively stop the algorithm and allow a restart or a reset. The current version of the software might lead to overflow errors whose effects are not known. A start/reset logic must be incorporated. This includes using an interrupt channel. Optionally, the failure mode should be displayed to the user. Not all failure modes are currently covered. Two likely failure modes that must be resolved are:

- Electronics failure.

- Accelerometer failure.

\subsection{Results Summary}

As shown in Figures 4 and 5, the control system delivers the required performance improvements. Note that the spectrum analyzer $A / D$ range was reduced in the closed loop case to improve signal to noise. This accounts for the lower noise floor in the closed loop spectrum. Table 1 displays the first six harmonic frequencies. Note that this test had a controller identical to the one that produced Figures 4 and 5, but was done on a different day.

Frequency Closed/Open (RMS)

$\begin{array}{ll}1 \mathrm{~F} & -63.4 \mathrm{~dB} \\ 2 \mathrm{~F} & -39.9 \mathrm{~dB} \\ 3 \mathrm{~F} & -45.1 \mathrm{~dB} \\ 4 \mathrm{~F} & -33.5 \mathrm{~dB} \\ 5 \mathrm{~F} & -36.5 \mathrm{~dB} \\ 6 \mathrm{~F} & -25.2 \mathrm{~dB}\end{array}$

Table 1: Closed loop performance for first six harmonics 
The stability margin of the counterbalance mass mode at $68 \mathrm{~Hz}$ was reduced by increasing the $2 \mathrm{~F}$ convergence speed to unnecessarily fast values. With a convergence time of the order of 1 second, stability remains extremely satisfactory. No instability problems were uncovered due to the $1 \mathrm{~F}$ filter. We noted, however, that harmonics $8 \mathrm{~F}$, $9 \mathrm{~F}$ and $10 \mathrm{~F}$ converge extremely slowly, the $8 \mathrm{~F}$ even being unstable. This indicates a poor knowledge of the phase of the loop transfer function. Stability of the $8 \mathrm{~F}$ filter was recovered by adding 180 degree to the transfer function. The first fix is to account for the phase delay due to the averaging. A second approach is to identify the overall loop transfer function including the control system and adjust the gains $\mathrm{g} \mathrm{I}_{\mathrm{n}}$ and $\mathrm{g} \mathrm{0}_{\mathrm{n}}$ to obtain 0 degree of phase at $1 F, 2 F$, etc. The procedure we used (besides the unaccounted time delay) is, however, very precise, as long as the plant transfer does not vary with the operating conditions. The instability of the $8 \mathrm{~F}$ filter indicates a possible migration of a pole-zero pair around $450 \mathrm{~Hz}$ large enough to create a destabilizing phase shift. By implementing the temperature control loop and by identifying the plant once again under controlled operating conditions, better phase information will be obtained and the convergence of the $8 \mathrm{~F}, 9 \mathrm{~F}$ and $10 \mathrm{~F}$ filters are very likely to improve. It might be necessary to gain schedule the LMS as well if the phase appears to vary too much with operating temperature. Because the compressor dynamics are highly non-linear, one should also consider the extreme case where the phase still varies with time and cannot be reliably estimated beforehand. If so, the control system should include an on-line identification routine which would periodically identify the varying phases and update the LMS filter gains accordingly. The necessity of this step is very unlikely, although the modifications are straightforward and could be easily accommodated on the current DSP chip.

\section{Summary of Procedure for New Cooler}

The existing system can be adapted for any similar cryocooler with a few additional steps. Many of the necessary parameters are calculated in either a MATLAB .m file or an Excel spreadsheet.

\subsection{Plant gain and phase}

The gain and phase at the harmonics is critical. The MATLAB file cryonot.m was used to correctly account for the phase and magnitude contributions of the notches. This code is very direct in MATLAB. An Excel spreadsheet is being developed to do the same thing.

The accelerometer input is notched at frequencies of local modes. In the code, three notches are implemented by a series of three two-state linear systems. The variables describing this acceleration conditioning, A11, A12, etc., are determined using the MATLAB script cryonot.m. Inputs to this file include the sampling rate and the frequencies of the notches. One of the notches is applied to the fundamental bounce mode frequency. The others are applied to potentially troublesome local modes (e.g. at 300 and $460 \mathrm{~Hz}$ ). An Excel file could be written to replace the MATLAB script.

Procedure for new cooler: 
Measure plant transfer function. Use an analog amplifier and the real accelerometer conditioning. Take the transfer function from the amplifier input to the accelerometer conditioning output. Make sure the amplifier response is flat and has no phase loss out to the highest frequency of interest (e.g. $\backslash 10$ times the fundamental). The main purpose of this test is to determine the magnitude and phase at the harmonics. The frequency of the main bounce mode and any local modes may also be determined. If these contribute sufficient phase near the harmonics frequencies they may need to be notched

Measure the gain of the amplifier in V/V by recording the RMS input and output - at the fundamental drive frequency.

\subsection{Calibrations}

The value BusPerADC should only have to be calibrated once. This represents the conversion from bus voltage to the computer's internal variable representing bus voltage. The surest way to find this number is to temporarily disable the toggling between voltage and temperature measurements. Setting LedOut $=0$; near the beginning of the interrupt service routine, just before the line set pwm(LED,LedOut);, will accomplish this. Then the multiplexer will not switch. The output of the multiplexer should then be recorded. Alternatively, the multiplexer input corresponding to buus voltage could be recorded. This will not however account for the loading from the IMI A/D input stage and a small error will be introduced. In either case

$$
\text { BusPerADC }=10\left(\frac{\text { Railvoltage }}{\text { Muxoutputvoltage }}\right)
$$

A typical value for BusPerADC is 84.5.

Accelerometer. This is a recording of the sensitivity of the accelerometer(s) used, including the signal conditioning. It is not absolutely critical to get this value exactly right. The algorithm will always attempt to minimize the acceleration. The calibration is important for, one accelerometer

$$
\text { ADCperG }=0.1 \text { (Accelerometer Volts } / g) \text {. }
$$

For two accelerometers whose charges are added,

$$
A D C \text { perG }=2 \text { times } 0.1 \text { (Accelerometer Volts/g.) }
$$

A typical value for $A D C p e r G$ is 0.2 .

Set nominal bus voltage. The variable PwrPerADC is simply the nominal bus voltage divided by 2048 . For $24 \mathrm{~V}$, PwrperADC $=0.0117188$.

Temperature. Two values need to be recorded: the offset and the gain factor. It is preferable, though not necessary, to set ledOut $=512$; near the beginning of the code, just before the line set pwm(LED,LedOut ); , before doing this calibration. Then the 
output of the mux will not switch from temperature to bus voltage, and the voltage corresponding to temperature will include the loading effect of the $A / D$ input.

Measuring the voltage correponding to temperature on the way into the mux will give a value that is very close.

The offset is determined by using an independent temperature measurement to confirm the system is at $77 \mathrm{~K}$. The value of Voffset $T$ should be set to $1 / 10$ of the voltage into the $A / D$. The assumption is that the $A / D$ has gain 0.1

The gain value Kper $A D C$ is determined using two temperature measurements. One near room temperature and one near $77 \mathrm{~K}$ will work. With an independent sensor used to confirm the temperature, the values $V_{A}$ and $V_{B}$ should be recorded at temperatures $T_{A}$ and $T_{B}$. Then KperADC is

$$
\operatorname{KperADC}=10 \frac{T_{A}-T_{B}}{V_{A}-V_{B}}
$$

Again, an A/D gain of 0.1 is assumed. A typical value for $K p e r A D C$ is 587 , which means that $1 \mathrm{~V}$ measured before the $\mathrm{A} / \mathrm{D}$ corresponds to $58.7 \mathrm{~K}$. 